\title{
Low Resolution Brain Source Localization Using EEG Signals
}

\author{
Munsif Ali Jatoi ${ }^{1}$, Nidal Kamel ${ }^{2}$, Syed Hyder Abbas Musavi ${ }^{1}$, \\ Muhammad Suhail Shaikh ${ }^{1}$ and Chandar Kumar ${ }^{1}$ \\ ${ }^{1}$ Faculty of Engineering, Science and Technology, Indus University, Karachi, \\ Sindh, Pakistan \\ ${ }^{2}$ Department of Electrical and Electronic Engineering, Universiti Teknologi \\ PETRONAS, Perak, Malaysia \\ munsif.jatoi@gmail.com
}

\begin{abstract}
Each mental or physical task gives rise to generate electromagnetic activity in the brain. These electrical signals are analyzed by using various neuroimaging techniques which include electroencephalography (EEG), magnetoencephalogy (MEG), positron emission tomography (PET) and functional magnetic resonance imaging (fMRI). However, when the brain sources which are responsible for such electrical activity are localized, then it's called brain source localization or source estimation. This information is utilized to comprehend brain's physiological, pathological, mental, functional abnormalities. Also, the information is used to diagnose cognitive behaviour of the brain. Various methodologies based upon EEG signals are adopted to localize the active sources such as minimum norm estimation (MNE), low resolution brain electromagnetic tomography (LORETA), standardized LORETA, exact LORETA, multiple signal classification (MUSIC), focal under- determined system solution (FOCUSS) etc. This research discusses localizing ability of low resolution techniques (LORETA and sLORETA) for various head models (finite difference model and concentric model). The simulations are carried out by using NETSTATION software. The results are compared in terms of activations for same EEG data with the same stimulus provided to subjects. However, it is observed that the combination of finite difference method (FDM) with sLORETA produced best results in terms of source intensity level (nA). Hence, the combination of inverse method sLORETA with FDM produces better source localization.
\end{abstract}

Keywords: Electroencephalography, Inverse Problem, Finite Difference Method, LORETA, Standardized LORETA

\section{Introduction}

Electroencephalography (EEG) is the invasive/non-invasive functional neuroimaging technique which is used to quantify neural activity by taking into account the electrical signals produced with the brain using a set of electrodes placed on the scalp[1-4]. EEG signals based localization of active brain sources is called EEG source localization or EEG inverse problem as the source model is constructed with the data parameters available [5].This EEG based source modelling is used for various neuroscience applications [6]. Due to the multipurpose application of brain source localization in the field of applied surgery and clinical purposes, it has got the attentions from various factions of society such as neurosurgeons, biomedical engineers, signal/image processing researchers and experts etc. It is evident from the fact that more than 200 publications are produced so far in this area of research. The EEG inverse problem is categorized as severely ill-posed inverse more than one solution can fit into data. This can be elaborated

Received (June 27, 2017), Review Result (September 4, 2017), Accepted (September 8, 2017) 
as the unknown parameters (active sources) outnumber the known parameters (electrodes used) [7].

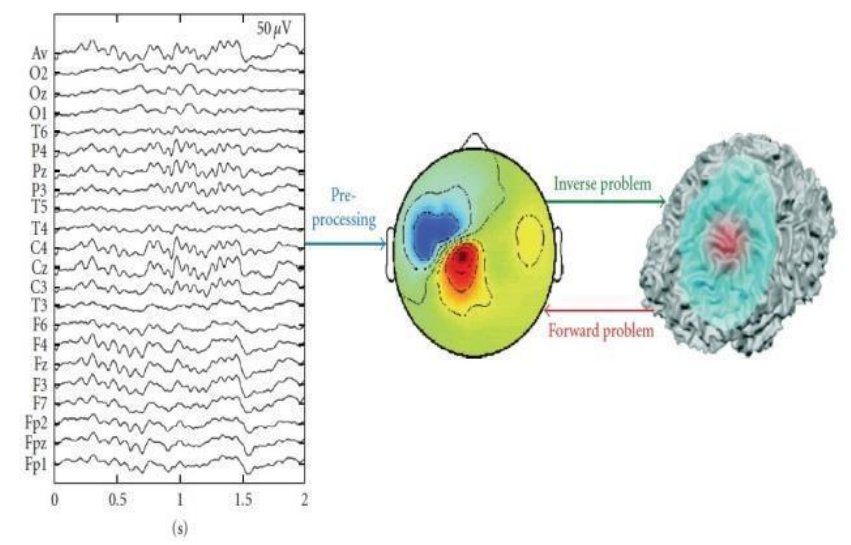

Figure 1. Inverse and Forward Problem Depiction[8]

Since inception of brain source localization issues, many techniques are suggested by various researchers to solve this ill-posed problem with better resolution, low computational complexity and low localization errors. However, the most significant contribution was made by minimum norm estimation (MNE) [9], low resolution brain electromagnetic tomography (LORETA) [10], standardized LORETA [11],exact LORETA[12], Multiple Signal classifier (MUSIC) and Recursively applied and projected MUSIC (RAP MUSIC)[13], focal under determined system solution (FOCUSS)[14], hybrid weighted minimum norm-LORETA (WMN-LORETA)[15], recursive sLORETAFOCUSS[16], standardized shrinking LORETA-FOCUSS (SSLOFO) [17], shrinking LORETA-FOCUSS[18] and Bayesian framework based multiple sparse priors[19-21]. This paper discusses low resolution techniques such as LORETA, sLORETA applied with numerical head modelling (Finite difference method) to produce the results for activation made during the data taken from subjects. This paper discusses low resolution techniques along with their implementation with various head modelling schemes. So here a brief account of LORETA and SLORETA is provided.

\section{LORETA}

This technique is elaborated and explained by R.D. Pascual-Marqui in [10]. LORETA estimates the active brain sources by calculating the distribution of current sources through the entire brain volume. This technique was proposed to localize the 3D solutions properly as compared to previous minimum norm estimation (MNE) approaches where there was no prior knowledge. However, LORETA imposes a spatial smoothness constraint upon solution. This spatial smoothness constraint is expressed using the 3D discretized Laplacian matrix as can be seen in literature [10]. Thus, LORETA estimates the sources with improved time resolution but low spatial resolution. For the low noise instantaneous measurements, the discrete solution for LORETA is derived as:

$$
\underset{J}{\min \|B W J\|^{2}} \quad \text { under constraint } \mathrm{Y}=\mathrm{LJ}
$$

where $Y \in \mathfrak{R}^{N_{c} \times N_{n}}$ is data captured by $N_{c}$ sensors and the number of time samples is $N_{n}$. The current density $J \in \mathfrak{R}^{N_{d} \times N_{n}}$ is responsible for the propagation of the energy of $N_{d}$ current dipoles distributed through the cortical surface. However, the dataset (Y) and the sources $(\mathrm{J})$ are related through gain matrix $\mathrm{L}$ which is also termed as leadfield matrix. 
Weighted matrix W is defined as: $W=\Omega \otimes I, \mathrm{I} \in \mathfrak{R}^{3 \times 3}$ is identity matrix and $\otimes_{\text {defines }}$ the Kronecker product, $\Omega$ is a diagonal matrix for which the diagonal element is defined by:

$$
\Omega_{i i}=\sqrt{\sum_{\alpha=1}^{N} L_{\alpha i}^{T} L_{\alpha i}}
$$

The discrete Laplace operator B is introduced to emphasize relationships between current densities and thus, the spatial resolution is not taken into consideration which makes the blurred localization images. For a regular cubic grid within the brain volume, if the distance between neighboring grids is assumed to be $\mathrm{d}$, then this operator is defined as:

$$
B=\frac{6}{d^{2}}\left(A-I_{3 M}\right)
$$

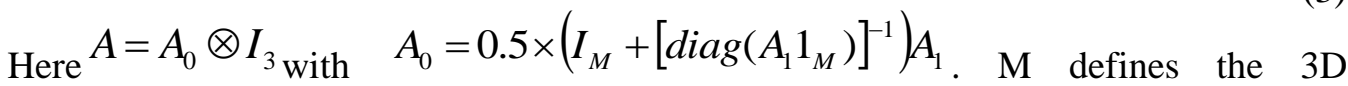
locations for the current densities. There are some modifications done on this basic localizing technique which include sLORETA, eLORETA and some hybrid algorithms such as Shrinking LORETA-FOCUSS, Standardized Shrinking LORETA-FOCUSS, WMN-LORETA, Recursive sLORETA-FOCUSS etc.

\section{SLORETA}

The initial variation on basic LORETA produced sLORETA as defined in [11]. sLORETA is based on standardization of current density supposed to be estimated for source localization. Hence, the current density estimate is carried out by using MNE approach and further it is standardized utilizing the expected standard deviation associated with it. The standard deviation is assumed to be created by noise exclusively. sLORETA is similar to method proposed by Dale [22] as it provides current density estimates by using MNE solution with localization inference computed through standardized current density. The way sLORETA adopts the standardization for current density is different and thus result is lesser localization error as compared to Dale method. As per standard definition for minimum norm estimates, the solution provide by it is harmonic in nature which implies that Laplacian of current density is zero (i.e. $\nabla^{2} J(r) \equiv 0$ ), where $\mathrm{r}$ denotes the volume coordinates in the brain. Thus, producing smooth solution as mentioned in MNE section above. Due to this, the deep point sources in the brain are not properly localized and localization error is relatively high. This issue can be resolved through standardization of MNE solution. Using same definitions for the variables as mentioned in previous methods, the functional of interest is given below:

$$
F=\|Y-L J-c\|^{2}+\alpha\|J\|^{2}
$$

Here $\alpha \geq 0$ is regularization parameter. This functional is supposed to be minimized with respected to $\mathrm{J}$ and $\mathrm{c}$ for given leadfield (L), voltage measurements (Y) and regularization parameter $(\alpha)$. The explicit solution for such minimization will be:

$$
J^{\prime}=T Y,{ }_{\text {with }} T=L^{T} H\left[H L L^{T} H+\alpha H\right]^{+}
$$

where $H \in \mathfrak{R}^{N_{E} \times N_{E}}$ is centering matrix and is defined as:

$$
H=I-\frac{11^{T}}{1^{T} 1}
$$

However, $I \in \mathfrak{R}^{N_{E} \times N_{E}}$ is identity matrix and $1 \in \mathfrak{R}^{N_{E} \times 1}$ is a vector of ones.

By using average reference for the measurements, one will have the functional as: 


$$
F=\|Y-L J\|^{2}+\alpha\|J\|^{2}
$$

With solution as:

$$
J^{\prime}=T Y,{ }_{\text {with }} T=L^{T}\left[L L^{T}+\alpha H\right]^{+}
$$

Hence, the estimated current density for SLORETA is given by:

$$
J^{\prime}=T L J=L^{T}\left(L L^{T}+\alpha H\right)^{+} L J=S_{J} J
$$

Here $S_{J^{\prime}}$ is variance of estimated current density.

In this way the sources are estimated in presence and absence of noise. This method has been used extensively for source localization with various head modeling schemes. Different neuroimaging software implements this technique which includes Netstation and LORETA etc. There are number of publications which followed sLORETA technique to localize the sources[23, 24]. The disadvantage with sLORETA is its low resolution which is due to regularization in solution for stability.

After discussion of low resolution methods in detail, now the related methodology is discussed for this research. Section II discusses methodology; Section III provides results and discussion. Section IV is dedicated for conclusion.

\section{Methodology}

Subjects

The data is collected by using proper ethics approval and consent from various healthy subjects. All of them are healthy, drug free and having no previous record of having any neurological disorder. All of them are currently university students between the ages of 20-30 years and with normal corrected vision.

Ethical Approval and Consent

The experimental details were provided to participants and they were signed before the start of the experiment. The Human Ethics Committee of Universiti Teknologi PETRONAS, Perak, Malaysia has approved the work. EEG data were captured at scalp surface with 250 samples per second using HydroCel Geodesic 128 channel montage and $300 \mathrm{Net}$ Amplifier of EGI Inc, USA [25] and Cz channel is taken as reference channel. The impedance between scalp and sensors was $50 \mathrm{~K} \Omega$. The EEG dense electrode sensor net is shown in Figure 02. The EEG recordings were done at Intelligent Neuro-Signals and Medical Imaging Laboratory, Centre for Intelligent Signals and Imaging Research (CISIR), Universiti Teknologi PETRONAS, Perak, Malaysia.

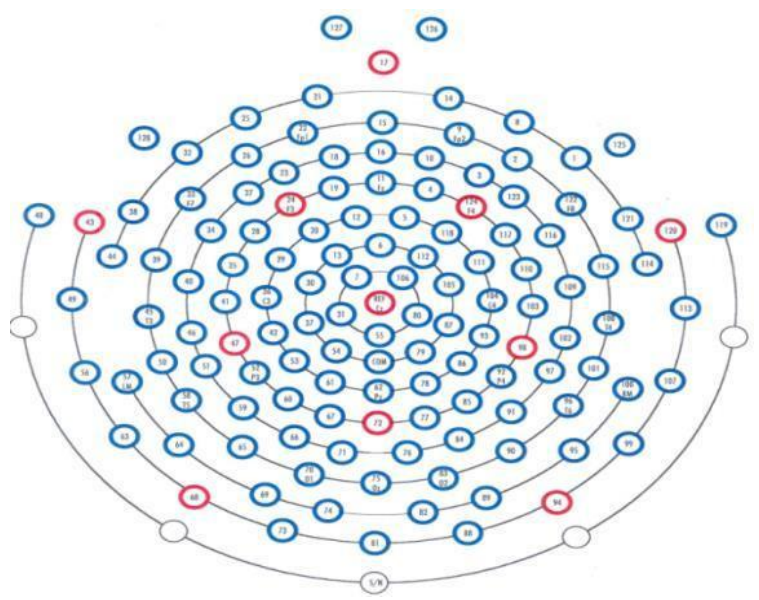

Figure 2. EEG Cap Layout 


\section{EEG Analysis}

The data acquired was subjected to filtering done by a band pass filter having frequency range as $1-48 \mathrm{~Hz}$. Prior to application of inverse methods such as LORETA, sLORETA, head modelling schemes (such as Finite difference method and concentric head model) was used for every set of data. After the application of head modelling, both of the inverse techniques are used to see the activation in the corresponding areas and to check the difference between them. The results are compiled for all cases to offer a comparison between various localization techniques with different modelling techniques. The Tikhonov regularization is applied with value of $1 \quad 10 \quad 4$.

\section{Results and Discussion}

The results showing active brain activation in certain parts are produced for subjects with specified head models and low resolution inverse techniques (LORETA and sLORETA) scheme and stated stimulus provided. Because in this research, only emphasize is given to low resolution methods, therefore the related results for the activation are provided for LORETA and sLORETA only. All the graphs are provided in 3 views i.e. sagittal, coronal and axial along with the activation region shown below. The color scheme is provided with the software accordingly. Due to ethical approval, the identity of the subjects is omitted. The results illustrate the activation in different brain during visual stimulus experiment. For both subjects, the results are collected for four different combinations of forward and inverse modelling. The combinations of head models and low resolution inverse methods are: 1) Sunstok-LORETA 2) SunstokSLORETA 3) FDM-LORETA 4) and FDM-sLORETA respectively. These combinations are utilized with the specifications stated in the methodology section. Hence, For example, for the subject $\mathrm{X}$, the process to find out the related activation was done for all combinations. The corresponding activation is shown for all mentioned combinations. The yellow colour demonstrates maximized activity in a particular region. In this case, the most activated region is occipital region for each combination. However, the maximum intensity of $280 \mathrm{nA}$ is shown with FDM-sLORETA combination. However, lower values of intensities were found for Sunstok-LORETA (0.92nA), Sunstok-sLORETA (5.35nA) and FDM-LORETA (15.782nA) is shown in Figure 3 and Figure 4 respectively. The similar trend was observed for the subject $\mathrm{Y}$ when it was tested through all possible combinations. The region which was most active during the given stimulus for subject $\mathrm{Y}$ is parietal region. However, the highest activation was observed for subject y was with FDM-sLORETA (61.82 nA). Other combinations have got lesser intensities as compared to FDM-sLORETA. Such as LORETA (0.279 nA), sLORETA (2.089nA) and FDMLORETA (7.40nA) respectively. The activation maps are produced in Figure 5 and Figure 6 respectively. These activation data with each combination for both subjects are produced in Table 01 and Table 02 given at the end of the article. Hence, the maximum value due to FDM-sLORETA is quite higher as compared to other combinations for both subjects. It is so because of high performance of sLORETA as compared with its older version LORETA. Although both of these methods are low resolution methods and have disadvantage of spatial blurring. But upon the analysis, it is clear that sLORETA exhibits less localization error and more intensive values for active regions when it was observed for same data with same stimulus provided. Another point worth to be to be noted from the above results is higher values of intensities for both subjects when the numerical head modeling (FDM) was applied. Hence, it can be seen from the Table 01 and Table 02 that significant difference can be observed between the activation intensities for both subjects. For example, for subject X, the intensities shown for FDM-LORETA and FDMsLORETA are $15.782 \mathrm{nA}$ and $280 \mathrm{nA}$. However, the intensities for same subject by using Sunstok-LORETA and Sunstok-sLORETA are $0.92 \mathrm{nA}$ and $5.35 \mathrm{nA}$ respectively. This fact can also be seen by looking into resultant activations for subject $\mathrm{Y}$ for numerical 
FDM-LORETA and FDM-sLORETA (7.40 nA and $61.82 \mathrm{nA}$ ) which is much higher than Sunstok-LORETA and Sunstok-sLORETA (0.279 nA and $2.089 \mathrm{nA})$ respectively. Hence, it is evident that numerical methods for head modelling such as boundary element method (BEM), finite element method (FEM) and finite difference method (FDM) out-performs the analytical methods (like Sunstok) which takes into account the nested shape concentric models for head modelling.
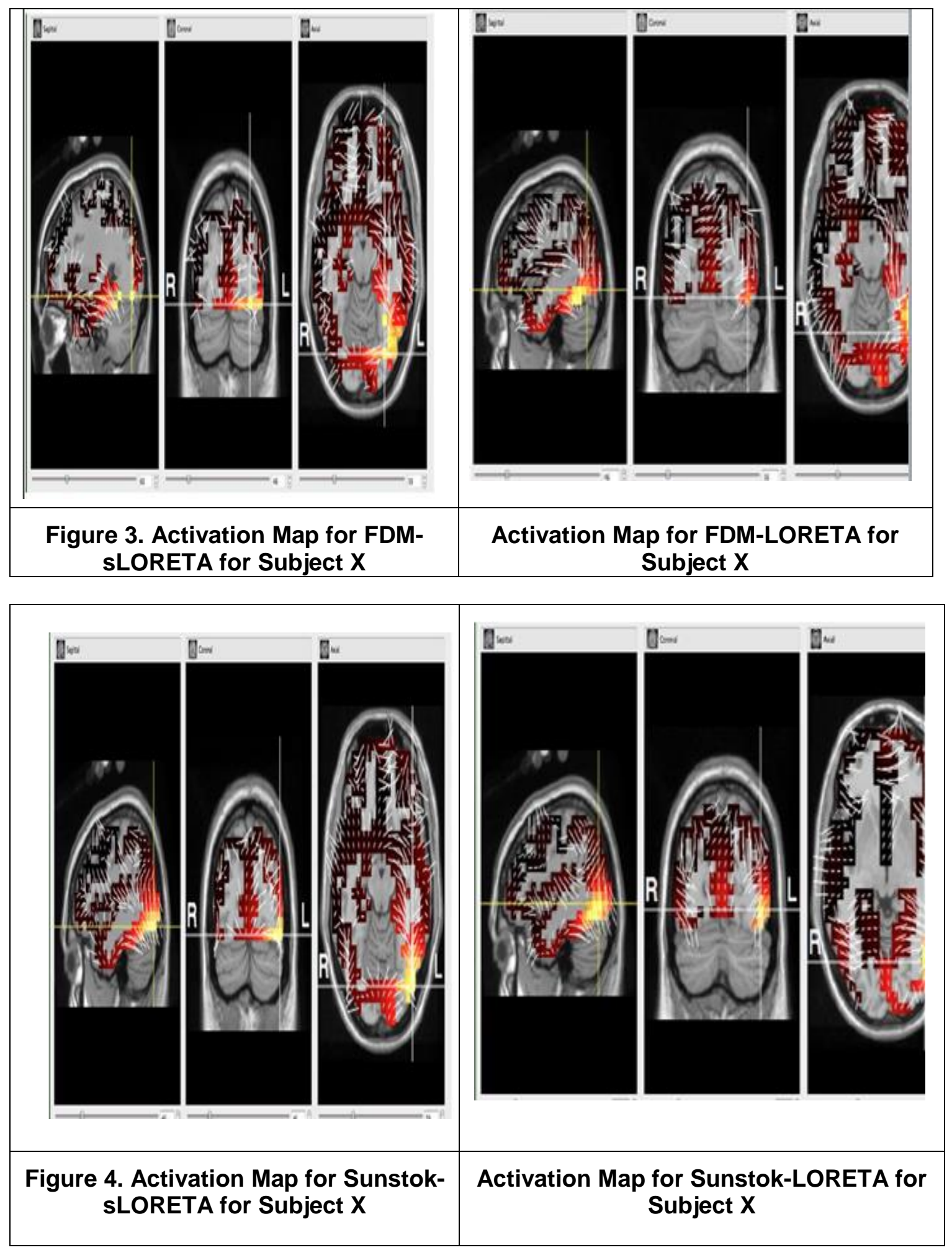


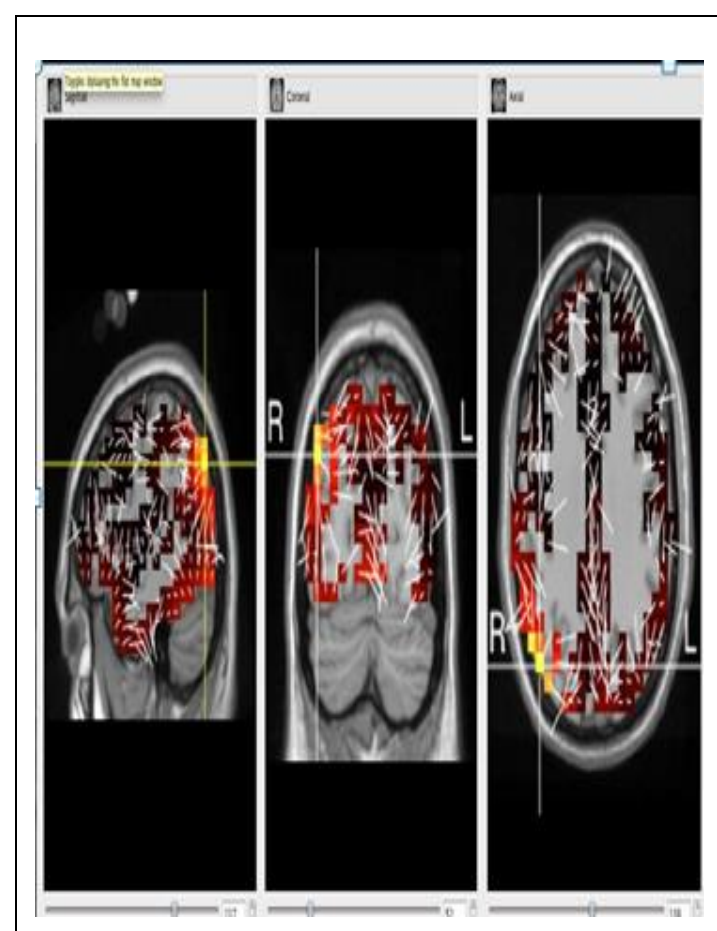

Figure 5. Activation Map for FDMsLORETA for Subject $Y$

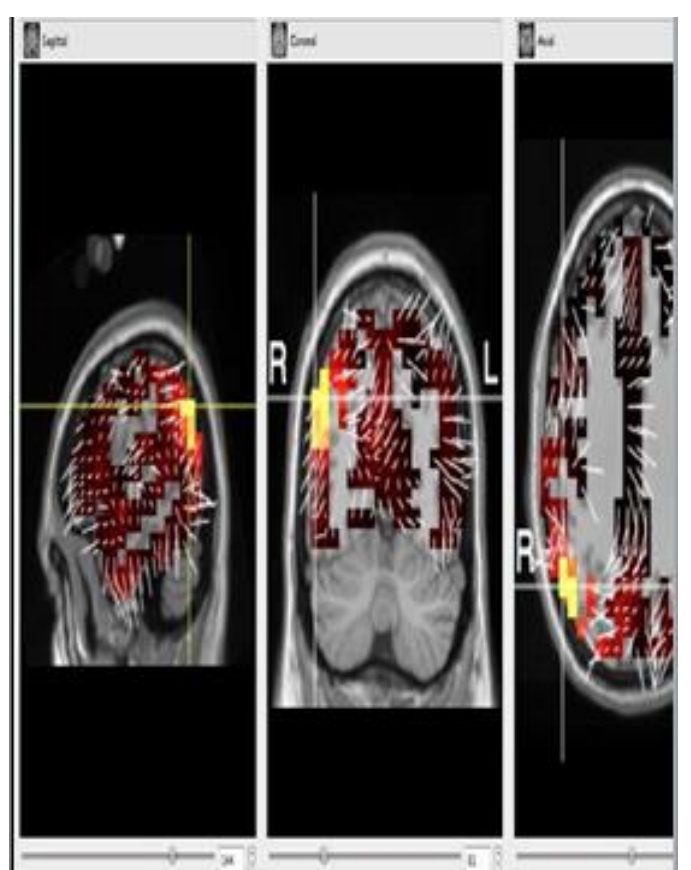

Activation Map for FDM-LORETA for Subject $Y$

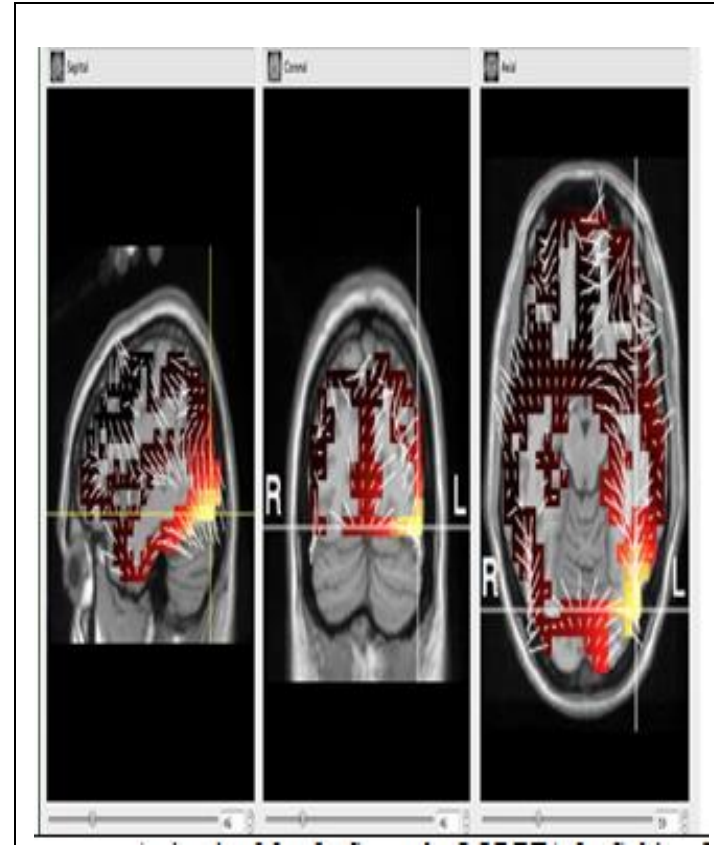

Figure 6. Activation Map for Sunstok-sLORETA for Subject $X$

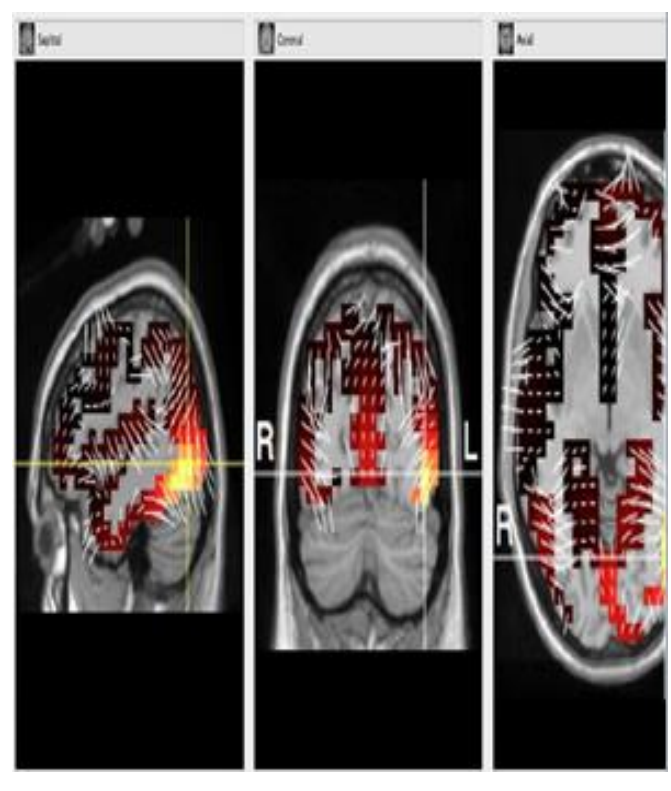

Activation Map for Sunstok-LORETA for Subject X 
Table 1. Activation Data for Subject $X$

\begin{tabular}{|l|l|l|l|}
\hline Subject & Method & Maximum Active Region & Intensity (nA) \\
\hline 1 & Sun Stok LORETA & Occipital Lobe & 0.92 \\
\hline 2 & Sun Stok -sLORETA & Occipital Lobe & 5.35 \\
\hline 3 & FDM-LORETA & Occipital Lobe & 15.782 \\
\hline 4 & FDM-sLORETA & Occipital Lobe & 280 \\
\hline
\end{tabular}

Table 2. Activation Data for Subject Y

\begin{tabular}{|l|l|l|l|}
\hline Subject & Method & Maximum Active Region & Intensity (nA) \\
\hline 1 & Sun Stok LORETA & Parietal lobe & 0.279 \\
\hline 2 & Sun Stok -sLORETA & Parietal lobe & 2.089 \\
\hline 3 & FDM-LORETA & Parietal lobe & 7.40 \\
\hline 4 & FDM-sLORETA & Parietal lobe & 61.82 \\
\hline
\end{tabular}

\section{Conclusion}

This research work discusses EEG based brain source localization by using various forward and inverse models. The EEG data was captured by using visual stimuli and localization results were obtained by introducing various combinations of forward and inverse techniques with the help of NETSTATION software package. The results showed activation in different brain regions i.e. occipital and parietal with varying intensity levels (measured in $\mathrm{nA}$ ) for all forward and inverse models combinations. Maximum intensity value was observed for FDM-sLORETA combination. The usage of numerical methods such as finite difference method (FDM) produces better results as compared to analytical models. This supports the idea of implementation of boundary element method (BEM) and finite element method (FEM) for various datasets with different stimuli and corresponding activations can be checked by adopting same methodology. The results and corresponding discussion presented above suggests that these low resolution methods such as LORETA, sLORETA and eLORETA results can be compared with high resolution subspace based multiple signal classification (MUSIC), recursively applied and projected MUSIC (RAP MUSIC) and other methods with varying head models (numerical and analytical) to check the active brain localization with low localization error and maximum accuracy in localizing active sources. These results are well utilized for clinical purposes for diagnoses of different neural diseases such as epilepsy and tumour. Also the application of these results is equally useful for cognitive and behavioral analysis and for brain research applications.

\section{Acknowledgment}

The authors are grateful to Center for Intelligent Signal and Imaging Research (CISIR), Universiti Teknologi PETRONAS, Perak, Malaysia for giving required facilities to conduct this research work.

\section{References}

[1] J. Ebersole, "EEG voltage topography and dipole source modeling of epileptiform potentials", Current Practice of Clinical Electroencephalography. Philadelphia: Lippincott Williams and Wilkins, (2003), pp. 732-752.

[2] S. Sanei and J. A. Chambers, EEG signal processing: John Wiley \& Sons, (2013).

[3] M. A. Jatoi, N. Kamel, A. S. Malik, I. Faye and T. Begum, "A survey of methods used for source localization using EEG signals”, Biomedical Signal Processing and Control, vol. 11, (2014), pp. 42-52. 
[4] B. J. Roth, D. Ko, I. R. von Albertini-Carletti, D. Scaffidi and S. Sato, "Dipole Localization in Patients with Epilepsy Using the Realistic Shaped Head Model", Electroencephalography and Clinical Neurophysiology, vol. 102, (1997).

[5] M. A. Jatoi, N. Kamel, A. S. Malik, I. Faye and T. Begum, "Representing EEG source localization using finite element method", in Control System, Computing and Engineering (ICCSCE), 2013 IEEE International Conference on, (2013), pp. 168-172.

[6] C. Plummer, A. S. Harvey and M. Cook, "EEG source localization in focal epilepsy: where are we now?", Epilepsia, vol. 49, (2008), pp. 201-218.

[7] A. Tarantola, "Inverse problem theory and methods for model parameter estimation", SIAM, (2005).

[8] K. Wendel, O. Väisänen, J. Malmivuo, N. G. Gencer, B. Vanrumste and P. Durka, "EEG/MEG source imaging: methods, challenges, and open issues", Computational intelligence and neuroscience, vol. 2009, (2009), p. 13.

[9] M. S. Hämäläinen and R. J. Ilmoniemi, "Interpreting magnetic fields of the brain: minimum norm estimates", Medical and biological engineering and computing, vol. 32, (1994), pp. 35-42.

[10] R. D. Pascual-Marqui, C. M. Michel and D. Lehmann, "Low resolution electromagnetic tomography: a new method for localizing electrical activity in the brain", International Journal of psychophysiology, vol. 18, (1994), pp. 49-65.

[11] R. D. Pascual-Marqui, "Standardized low-resolution brain electromagnetic tomography (sLORETA): technical details", Methods Find Exp Clin Pharmacol, vol. 24, (2002), pp. 5-12.

[12] R. D. Pascual-Marqui, "Discrete, 3D distributed, linear imaging methods of electric neuronal activity. Part 1: exact, zero error localization", arXiv preprint arXiv:0710.3341, (2007).

[13] J. C. Mosher and R. M. Leahy, "Source localization using recursively applied and projected (RAP) MUSIC", IEEE Transactions on signal processing, vol. 47, (1999), pp. 332-340.

[14] I. F. Gorodnitsky and B. D. Rao, "Sparse signal reconstruction from limited data using FOCUSS: A reweighted minimum norm algorithm", IEEE Transactions on signal processing, vol. 45, (1997), pp. 600616.

[15] C. Song, T. Zhuang and Q. Wu, "Hybrid weighted minimum norm method a new method based LORETA to solve EEG inverse problem", in Engineering in Medicine and Biology Society, 2005. IEEE-EMBS 2005. 27th Annual International Conference of the, (2006), pp. 1079-1082.

[16] R. Khemakkhem, W. Zouch, A. Taleb-Ahmed and A. B. Hamida, "A new combining approach to localizing the EEG activity in the brain: WMN and LORETA solution", in BioMedical Engineering and Informatics, 2008. BMEI 2008. International Conference on, (2008), pp. 821-824.

[17] H. Liu, P. H. Schimpf, G. Dong, X. Gao, F. Yang, and S. Gao, "Standardized shrinking LORETAFOCUSS (SSLOFO): a new algorithm for spatio-temporal EEG source reconstruction," IEEE Transactions on Biomedical Engineering, vol. 52, (2005), pp. 1681-1691.

[18] H. Liu, X. Gao, P. H. Schimpf, F. Yang, and S. Gao, "A recursive algorithm for the three-dimensional imaging of brain electric activity: shrinking LORETA-FOCUSS," IEEE Transactions on Biomedical Engineering, vol. 51, (2004), pp. 1794-1802.

[19] K. Friston, L. Harrison, J. Daunizeau, S. Kiebel, C. Phillips, N. Trujillo-Barreto,, "Multiple sparse priors for the M/EEG inverse problem," NeuroImage, vol. 39, (2008), pp. 1104-1120.

[20] M. A. Jatoi, N. Kamel, J. D. López, I. Faye, and A. S. Malik, "MSP based source localization using EEG signals," in Intelligent and Advanced Systems (ICIAS), 2016 6th International Conference, (2016), pp. $1-5$.

[21] J. López, V. Litvak, J. Espinosa, K. Friston, and G. R. Barnes, "Algorithmic procedures for Bayesian MEG/EEG source reconstruction in SPM," NeuroImage, vol. 84, (2014), pp. 476-487.

[22] A. M. Dale, A. K. Liu, B. R. Fischl, R. L. Buckner, J. W. Belliveau, J. D. Lewine., "Dynamic statistical parametric mapping: combining fMRI and MEG for high-resolution imaging of cortical activity," Neuron, vol. 26, (2000), pp. 55-67.

[23] M. A. Jatoi, N. Kamel, A. S. Malik, I. Faye, J. M. Bornot, and T. Begum, "EEG-based brain source localization using visual stimuli," International Journal of Imaging Systems and Technology, vol. 26, (2016), pp. 55-64.

[24] M. A. Jatoi, N. Kamel, A. S. Malik, and I. Faye, "EEG based brain source localization comparison of sLORETA and eLORETA," Australasian physical \& engineering sciences in medicine, vol. 37, (2014), pp. 713-721. 
International Journal of Signal Processing, Image Processing and Pattern Recognition Vol. 10, No. 10 (2017) 